\title{
Receptor-like protein tyrosine phosphatase $\kappa$ negatively regulates the apoptosis of prostate cancer cells via the JNK pathway
}

\author{
PING-HUI SUN, LIN YE, MALCOLM D. MASON and WEN G. JIANG \\ Metastasis and Angiogenesis Research Group, Institute of Cancer and Genetics, \\ Cardiff University School of Medicine, Cardiff CF14 4XN, UK
}

Received May 20, 2013; Accepted June 25, 2013

DOI: 10.3892/ijo.2013.2082

\begin{abstract}
Receptor-like protein tyrosine phosphatase $\kappa$ (PTPRK) has been indicated as a putative tumour suppressor in primary central nervous system lymphomas and colorectal cancer. The present study investigated the expression of PTPRK in prostate cancer and the biological impact of PTPRK on prostate cancer cells. The expression of the PTPRK protein and transcript in prostate cancer was examined using IHC and PCR. Knockdown of PTPRK in prostate cancer cells was performed using a specific anti-PTPRK transgene. The impact of PTPRK knockdown on prostate cancer cells was evaluated using in vitro cell models and the apoptosis was analysed using flow cytometry. PTPRK expression was increased in prostate cancer tissues and knockdown of PTPRK in PC-3 cells suppressed the in vitro cell growth in which an increased apoptotic population was seen. Accompanied with the knockdown of PTPRK, increased expression of caspase-3, caspase-8 and p53, and a decreased ID1 expression were evident in the cells. Furthermore, an increased tyrosine phosphorylated c-Jun N-terminal kinase (JNK) was seen in the PTPRK knockdown cells. The effect on apoptosis was diminished by a JNK inhibitor. In conclusion, PTPRK knockdown resulted in increased apoptosis leading to the inhibition of in vitro growth of prostate cancer cells. PTPRK is a key factor in coordinating apoptosis via the regulation of MAPK pathways, in particular the JNK pathway in prostate cancer cells.
\end{abstract}

\section{Introduction}

Apoptosis, also known as programmed cell death, can be induced by both intrinsic and extrinsic pathways. The intrinsic pathway is also known as mitochondria pathway and is regulated by different molecules such as XIAP (inhibitors of apoptosis) and Bcl-2 family. The extrinsic pathway is activated by tumour

Correspondence to: Dr Ping-Hui Sun, Metastasis and Angiogenesis Research Group, Institute of Cancer and Genetics, Cardiff University School of Medicine, Cardiff CF14 4XN, UK

E-mail: sunp2@cf.ac.uk

Key words: receptor-like protein tyrosine phosphatase $\kappa$, prostate cancer, apoptosis, c-Jun N-terminal kinase necrosis factors (TNFs) and its down-stream transcription factors are also involved in intrinsic pathway of apoptosis. The extrinsic pathway involves the promotion of apoptosis via the ligand-activation of death receptors (1). For example, TNF- $\alpha$ binding to its receptors leads to form two complexes; one can lead to activation of pro-survival NF- $\mathrm{B}$ pathway and another one activates the apoptosis pathway through Fas associated death domain (FADD) and activation of caspase-8 (2). Protein tyrosine phosphatases (PTPs) play crucial roles in regulation of cell functions such as proliferation and survival.PTPs deficiency leads to several physiologic abnormalities and dysregulation of apoptosis resulted by PTP deficiency may play a profound role in these disorders. For example, PTP-PEST (tyrosine-protein phosphatase non-receptor type 12, PTPN12) makes cells more sensitive to the anti-Fas and TNF- $\alpha$ induced apoptosis. PTP-PEST is cleaved by caspase-3, which increases its catalytic activity and changes its protein structure. Furthermore, the PTP-PEST proteolysis facilitates cellular detachment during apoptosis (3). Unlike PTP-PEST, PTP1B (PTPN1) has no caspase cleavage site and it is not cleaved by caspases during apoptosis. However, there is a report showing activated PTP1B contributes to STAT3 dephosphorylation and induces apoptosis in human glioma cells $(3,4)$. PTP1B and its catalytic activity are required for inositol-requiring kinase 1 (IRE1) signalling which activates JNK and p38 MAPK. Moreover, endoplasmic reticulum (ER)-induced apoptosis is decreased in cells lacking PTP1B and PTP1B null mice are resistant to Fas-induced liver damage because of absence of PTP1B-mediated suppression of pro-survival $\mathrm{NF}-\kappa \mathrm{B}$ and $\mathrm{ERK}$ signalling $(5,6)$. In addition, $\mathrm{p} 53$ is required for $\mathrm{T}$ cell protein tyrosine phosphatase (TC-PTP, PTPN2) overexpression induced apoptosis. In breast cancer cells, MCF-7, the accumulation of p53 in response to TC-PTP overexpression directly increases expression of Apaf-1 and pro-apoptotic $\alpha$-isoform of caspase-1 $(7,8)$.

Currently, the role played by PTPRK in cancer remains largely unknown. The present study examined the expression of PTPRK in prostate cancer and the impact of this molecule on prostate cancer cell apoptosis.

\section{Materials and methods}

Cell lines and cells culture. PC-3 (human prostate cancer cell line) and MRC-5 (fibroblast cell line) were obtained from the European Collection of Animal Cell Cultures (ECACC, 
Table I. Primer sequences used in the current study.

\begin{tabular}{lll}
\hline Molecular & \multicolumn{1}{c}{ Forward primers (5'-3') } & \multicolumn{1}{c}{ Reverse primers (5'-3') } \\
\hline PTPRK & AATTACAATTGATGGGGAGA & CCACTTTTCCACCTGAAGTA \\
PTPRK (Q-PCR) & AATTACAATTGATGGGGAGA & ACTGAACCTGACCGTACATATTGTGTGACGATGAAAGC \\
GAPDH & GGCTGCTTTTAACTCTGGTA & GACTGTGGTCATGAGTCCTT \\
GAPDH (Q-PCR) & CTGAGTACGTCGTGGAGTC & ACTGAACCTGACCGTACAGAGATGACCCTTTTG \\
c-Myc & TGCTCCATGAGGAGACAC & TTTCATTGTTTTCCAACTCC \\
c-Myc (Q-PCR) & TGCTCCATGAGGAGACAC & ACTGAACCTGACCGTACATGATCCAGACTCTGACCTTT \\
ID1 & TCAACGGCGAGATCAG & ACTGAACCTGACCGTACAGATCGTCCGCAGGAA \\
p53 & ATCCTCACCATCATCACACT & ACTGAACCTGACCGTACACAGGACAGGCACAAACAC \\
Caspase-3 & GGCGTGTCATAAAATACCAG & ACTGAACCTGACCGTACAACAAAGCGACTGGATGAA \\
Caspase-8 & AGAAAGGAGGAGATGGAAAG & ACTGAACCTGACCGTACAGACCTCAATTCTGATCTGCT \\
Caspase-9 & AAGCCCAAGCTCTTTTTC & ACTGAACCTGACCGTACAGTTACTGCCAGGGGACTC
\end{tabular}

Bold text indicates the specific $\mathrm{Z}$ sequence for Real-time PCR.

Salisbury, UK). DU-145, LNCaP, CA-HPV-10 and PZ-HPV-7 were obtained from the American Type Culture Collection (ATCC, Rockville, MD, USA) and PNT-1A and PNT-2C2 were generously given by Professor Norman Maitland (University of York, York, UK). Cells were routinely cultured with Dulbecco's modified Eagle's medium containing 10\% fetal calf serum and antibiotics at $37^{\circ} \mathrm{C}$ with $5 \% \mathrm{CO}_{2}$.

Human prostate specimens. Fresh tissue samples were collected immediately after surgery and stored at $-80^{\circ} \mathrm{C}$ until use, with approval of the Bro Taf Health Authority local research ethics committee. All patients were informed and participated with written consent. All the specimens were verified by a consultant pathologist.

Immumohistochemical staining. Frozen specimens of mammary tissues were cut at a thickness of $6 \mu \mathrm{m}$ using a cryostat (Leica CM 1900, Leica Microsystems UK Ltd., Buckinghamshire, UK). The sections were mounted on super frost plus microscope slides, air dried and then fixed in the mixture of 50\% acetone and 50\% methanol for $15 \mathrm{~min}$. After $10 \mathrm{~min}$ air-drying, the slides were placed into OptiMax Wash Buffer (BioGenex, San Ramon, CA, USA) for 5 min to rehydrate. The slides were incubated in blocking buffer with $10 \%$ horse serum for $20 \mathrm{~min}$ and probed anti-PTPRK antibody (Santa Cruz Biotechnology, Santa Cruz, CA, USA) for $1 \mathrm{~h}$. After three times washes, the slides were incubated with biotinylated secondary antibody (Multilink swine anti-goat/mouse/rabbit immunoglobulin, Dako Inc., Carpinteria, CA, USA). After washing, slides were placed in avidin-biotin complex (ABC, Vector Labs) for $30 \mathrm{~min}$. Diaminobenzidine chromogen (Vector Labs) was then added to the slides and incubated in the dark for $5 \mathrm{~min}$. The slides were counterstained with Mayer's haematoxylin for $1 \mathrm{~min}$ and dehydrated in ascending grades of ethanol before clearing in xylene and mounting with a cover slip.

Reverse transcription-PCR. Total RNA extraction from frozen tissues and culture cells was performed using Tri Reagent (Sigma-Aldrich Inc., Saint Louis, MO, USA). Following reverse transcription into cDNA, PCR was carried out using ReadyMix PCR Reaction Mix (Sigma-Aldrich Inc.). Primer sequences are shown in Table I. Reactions were carried out at the following conditions: $94^{\circ} \mathrm{C}$ for $5 \mathrm{~min}, 30$ cycles of $94^{\circ} \mathrm{C}$ for $30 \mathrm{sec}, 55^{\circ} \mathrm{C}$ for $30 \mathrm{sec}$ and $72^{\circ} \mathrm{C}$ for $30 \mathrm{sec}$, followed by a final extension of $7 \mathrm{~min}$ at $72^{\circ} \mathrm{C}$. PCR products were separated on a $1.5 \%$ agarose gel and photographed after staining with ethidium bromide.

Real-time quantitative PCR. The level of PTPRK transcripts in the breast cancer cohort was determined using a real-time quantitative PCR, based on technology which was modified from a method reported previously (9). Primer sequences are shown in Table I. The reaction was carried out on an iCycler $\mathrm{iQ}^{\mathrm{TM}}$ (Bio-Rad, Hertfordshire, UK) which is equipped with an optical unit that allows real-time detection of 96 reactions. The reaction conditions were: $94^{\circ} \mathrm{C}$ for $12 \mathrm{~min}, 90$ cycles of $94^{\circ} \mathrm{C}$ for $15 \mathrm{sec}, 55^{\circ} \mathrm{C}$ for $40 \mathrm{sec}$ (the data capture step) and $72^{\circ} \mathrm{C}$ for $20 \mathrm{sec}$. The levels of the transcripts were generated from an internal standard that was simultaneously amplified with the samples.

Construction of ribozyme transgene targeting human PTPRK and the establishment of corresponding stable transfectants. Anti-human PTPRK hammerhead ribozymes were designed based on the secondary structure of the gene transcript and generated using the Zuker RNA mFold program (10). The ribozymes were synthesized and then cloned into a pEF6/V5-His TOPO vector (Invitrogen, Paisley, UK). The verified ribozyme transgenes and empty plasmids were transfected into PC-3 (PC-3 $3^{\text {PTPRKd }}$ and PC- $\left.3^{\mathrm{PEF}}\right)$ cells, respectively, using an EasyjetPlus electroporator (EquiBio, Kent, UK). After a period of selection with $5 \mu \mathrm{g} / \mathrm{ml}$ blasticidin (up to 10 days), the verified transfectants were cultured in maintenance medium containing $0.5 \mu \mathrm{g} / \mathrm{ml}$ blasticidin. Primer sequences of the ribozymes were 5'-CTGCAGTTTGCTCTT TTTTACAATTAATATCTGATGAGTCCGTGAGGA-3' and 5'-ACTAGTTCATCCTCCTTCTCCTAGTTGTTTCGTCCT CACGGACT-3'. 
Table II. Primary antibodies used in the current study.

\begin{tabular}{ll}
\hline Protein target & Cat. no. \\
\hline Mouse anti-GAPDH & SC-47724 \\
Rabbit anti-PTPRK & SC-28906 \\
Mouse anti-p38 & SC-7972 \\
Rabbit anti-JNK & SC-571 \\
Rabbit anti-caspase-3 & SC-7148 \\
Mouse anti-caspase-8 & SC-70501 \\
Mouse anti-caspase-9 & SC-17784 \\
Mouse anti-phosphotyrosine & SC-508
\end{tabular}

Cell growth assay. Prostate cancer cells (3,000 cells/well) were plated into 96 -well plates. Cells were fixed in $4 \%$ formaldehyde after 1 and 5 days of culture (11). The cells were then stained with $0.5 \%$ crystal violet. Absorbance was determined at a wavelength of $540 \mathrm{~nm}$ using a spectrophotometer (BioTek, ELx800). Growth rate at day $5(\%)=$ absorbance of day $5 /$ absorbance of day $1 \times 100$.

Analysing apoptosis using flow cytometer. Cells $\left(1 \times 10^{5}\right)$ were seeded into flasks and underwent different treatments with $200 \mathrm{nM}$ p38 inhibitor (SB203580), $200 \mathrm{nM}$ JNK inhibitor (SP600125) and serum free control for three days then both the adherent cells and floating cells were collected. Following cell collection, experiments were carried out using the Annexin V kit (Santa Cruz Biotechnology) and analysed using PartecCyFlow ${ }^{\circledR}$ SL flow cytometry and the accompanying FloMax software package (Partec GmbH, Münster, Germany).

Immunoprecipitation (IP) and western blot analysis. Protein was extracted from $75-\mathrm{cm}^{2}$ flask which were initially seeded with $4 \times 10^{6}$ cells and cultured overnight. The protein samples were incubated with primary antibodies (Table II) at $4^{\circ} \mathrm{C}$ for $1 \mathrm{~h}$ then incubated for another hour after the addition of conjugated A/G protein agarose beads (Santa Cruz Biotechnology). The samples were washed twice with SDS-free lysis buffer before being boiled with $1 \mathrm{X}$ sample buffer (Sigma-Aldrich Inc.).

Equal amounts of protein were separated by SDS-PAGE and blotted onto nitrocellulose membranes. The membrane was then probed with the respective primary antibodies and corresponding peroxidase-conjugated secondary antibodies. Protein bands were visualised using a chemiluminescence detection kit (Luminata, Millipore) and photographed using UVITech imager (UVITech Inc.).

Statistical analysis. Statistical analysis was performed using SigmaPlot 11 (SPSS Inc., Chicago, IL, USA). Data were calculated as the mean \pm SD and the Student's t-test was used for normally distributed data. Fisher's exact test was used for comparison of two independent groups. Each assay was performed at least three times independently. $p<0.05$ was considered statistically significant.

\section{Results}

PTPRK expression in prostate tissues and cell lines. There is a higher PTPRK expression level in prostate cancer tissues compared with normal prostate tissues using IHC staining (Fig. 1A). Fig. 1B shows that PTPRK is likely to be more frequently expressed in prostate cancer tissues (12/18, 66.7\% positive, $\mathrm{p}=0.143$ vs. normal using Fisher's exact test) than normal prostate tissues at mRNA level (4/11, 36.7\% positive). Furthermore, the expression of PTPRK was also examined in the prostate cell lines PC-3, DU-145, LNCaP, CA-HPV-10, PZ-HPV-7, PNT-1A, PNT-2C2 and the fibroblast cell line MRC-5. Fig. 1C shows that PTPRK is consistently expressed in all the prostate cell lines, except PNT-1A, where it appears to have a lower level of PTPRK mRNA.

Verification of PTPRK knockdown in PC-3 cells. The expression of PTPRK was knocked down using ribozyme transgenes targeting human PTPRK mRNA. This was performed in the prostate cancer cell line PC-3, which expressed PTPRK (Fig. 1C). The knockdown of PTPRK was verified in the transfectants using RT-PCR (Fig. 2A), real-time quantitative PCR (Fig. 2B), western blot analysis (Fig. 2C) and PTPRK protein band volume of three repeats which was normalised against corresponding internal control (Fig. 2D). Decreased expression of PTPRK was seen in PC-3 $3^{\text {PTPRKkd }}$ cells which were transfected with ribozyme transgenes compared to their corresponding empty plasmid control.

Knockdown of PTPRK reduces in vitro cell growth assay. Knockdown of PTPRK in PC-3 cells exhibited an impact on cell growth. The PC-3 $3^{\text {PTPRKkd }}$ cells showed a decreasing growth rate at day $5(634.33 \pm 58.76, \mathrm{p}<0.001)$ compared with PC-3 $3^{\mathrm{EF}}$ (739.35 \pm 24.14$)$ (Fig. 3A).

PTPRK knockdown affects apoptosis in prostate cancer cells. There was an increased proportion of apoptotic cells (both early and late) in PC-3 $3^{\text {PTPRKkd }}(34.90 \%)$ compared to the PC-3 ${ }^{\mathrm{pEF}}$ control (16.14\%) and PC-3 wild-type (8.05\%) cells (Fig. 3B).

Expression of caspases in the PTPRK knockdown cells. Caspase- 3 is an indicator of apoptosis at the end-stage. Caspase- 8 and caspase- 9 are up-stream key factors of apoptosis; caspase- 8 is normally activated by external signalling, and caspase- 9 is activated by internal signalling. Therefore, in order to further determine whether PTPRK knockdown has an effect on prostate cancer cell apoptosis, levels of caspase-3, -8 and -9 were examined in the transfected PC-3 cells using PCR, Q-PCR, and western blot analysis. PC-3 $3^{\text {PTPRKkd }}$ cells demonstrated significantly higher expression levels of caspase- 3 and caspase- 8 , but not caspase-9, compared with PC- $3^{\mathrm{pEF}}$ controls cells in both mRNA and protein levels (Fig. 4).

Expression of other genes/molecules relevant to apoptosis and/or the cell cycle. As PTPRK knockdown was shown to promote the progression of apoptosis, the expression of a number of relevant genes was examined using RT-PCR. An upregulation of p53 was also seen in the PTPRK knockdown cells, whilst a downregulation of ID1 appeared in the same cells 
A

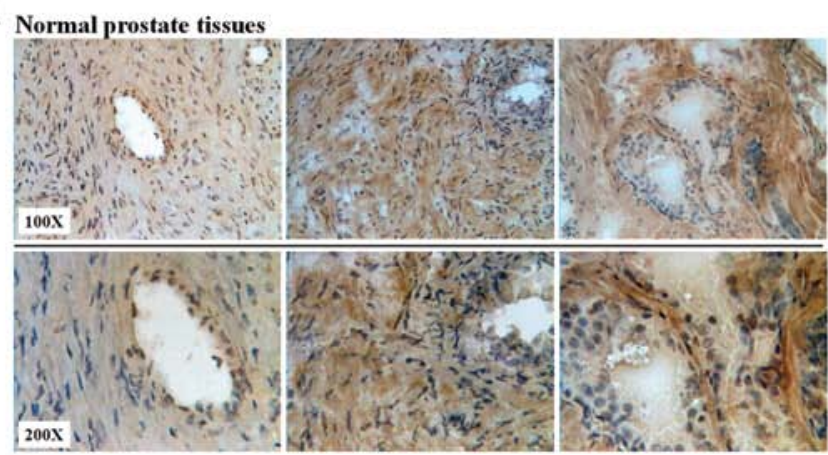

Prostate cancer tissues

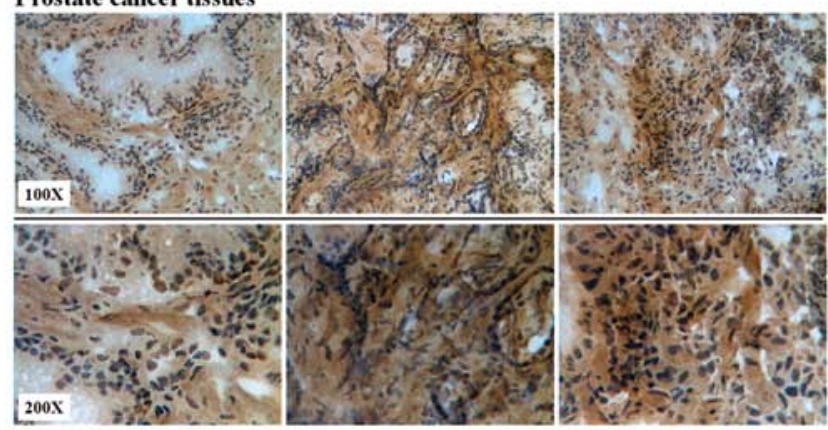

B

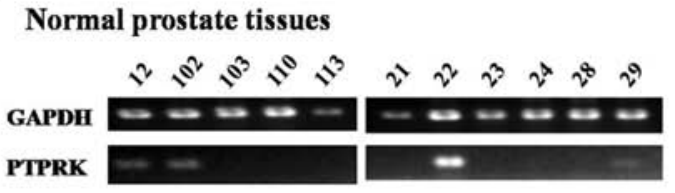

Prostate cancer tissues

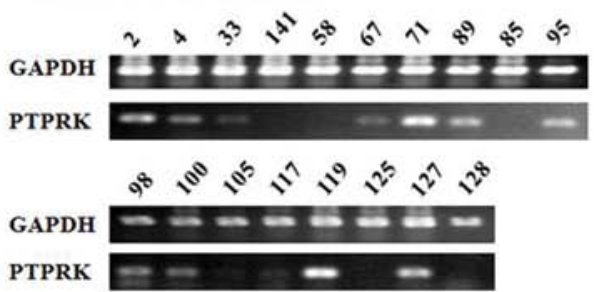

C

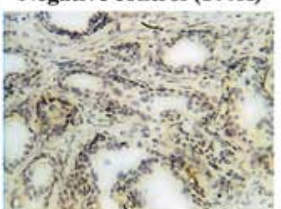

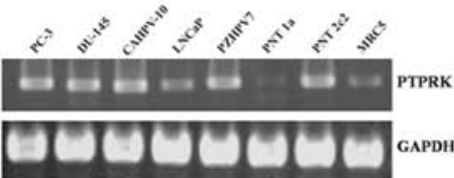

Figure 1. Expression of PTPRK in prostate cancer. (A) The immunohistochemical staining revealed an increase staining of PTPRK in prostate cancer tissues $(n=17)$ compared with normal tissues $(n=8)$. (B) PTPRK is more frequently expressed in prostate cancer tissues compared with normal tissues. (C) Expression of PTPRK mRNA in cell lines using RT-PCR.

A

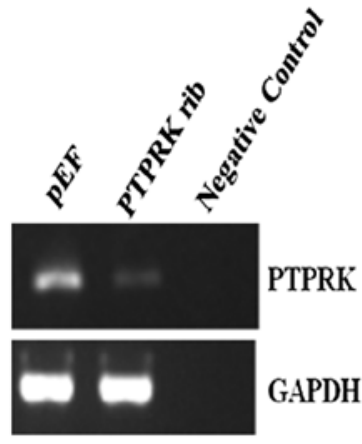

PC-3
B

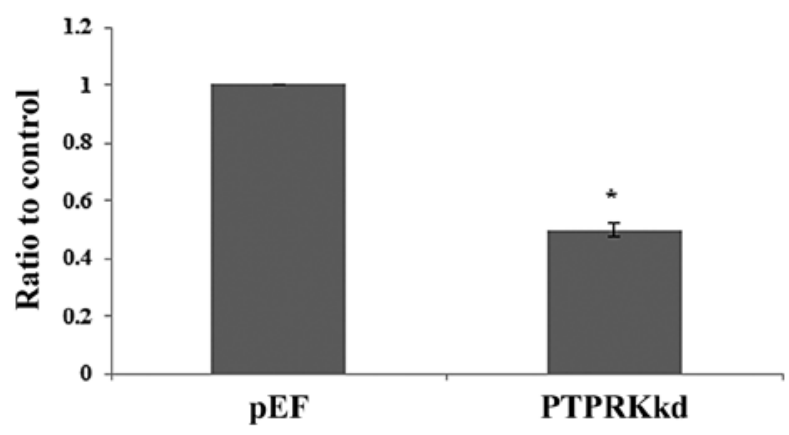

D

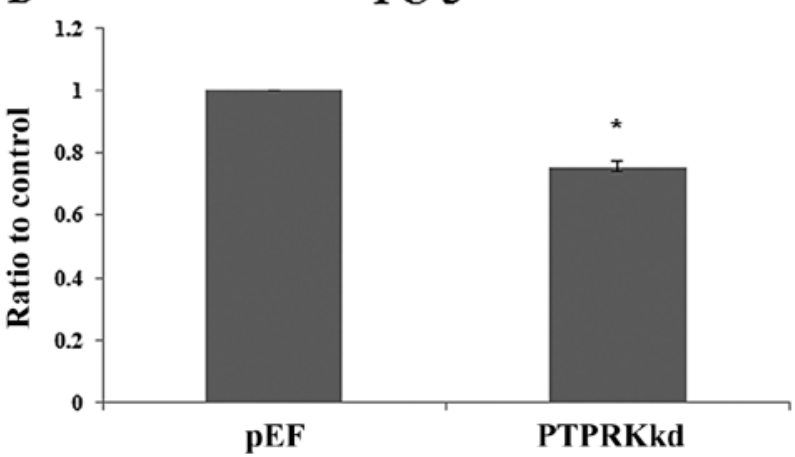

Figure 2. Confirmation of PTPRK knockdown in PC-3 cells. Knockdown of PTPRK was seen in PC-3 ${ }^{\text {PTPRKkd }}$ cells compared with empty plasmid control (PC-3 $3^{\text {pEF }}$ cells) using (A) RT-PCR, (B) real-time quantitative PCR and (C) western blot analysis. (D) PTPRK protein band volume of three repeats, which was normalised against corresponding internal control. ${ }^{*} \mathrm{p}<0.05$. 


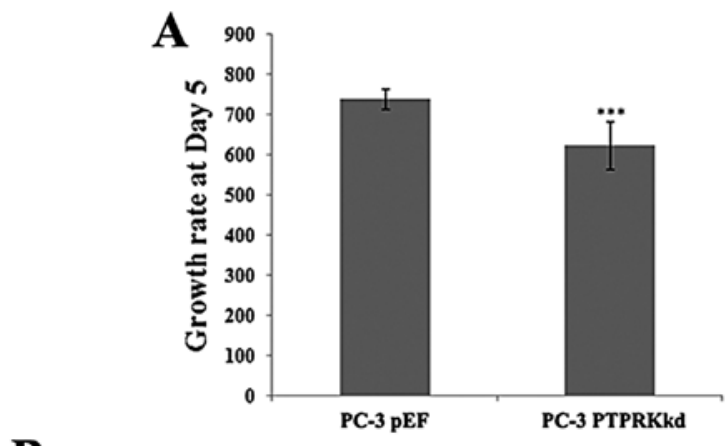

B

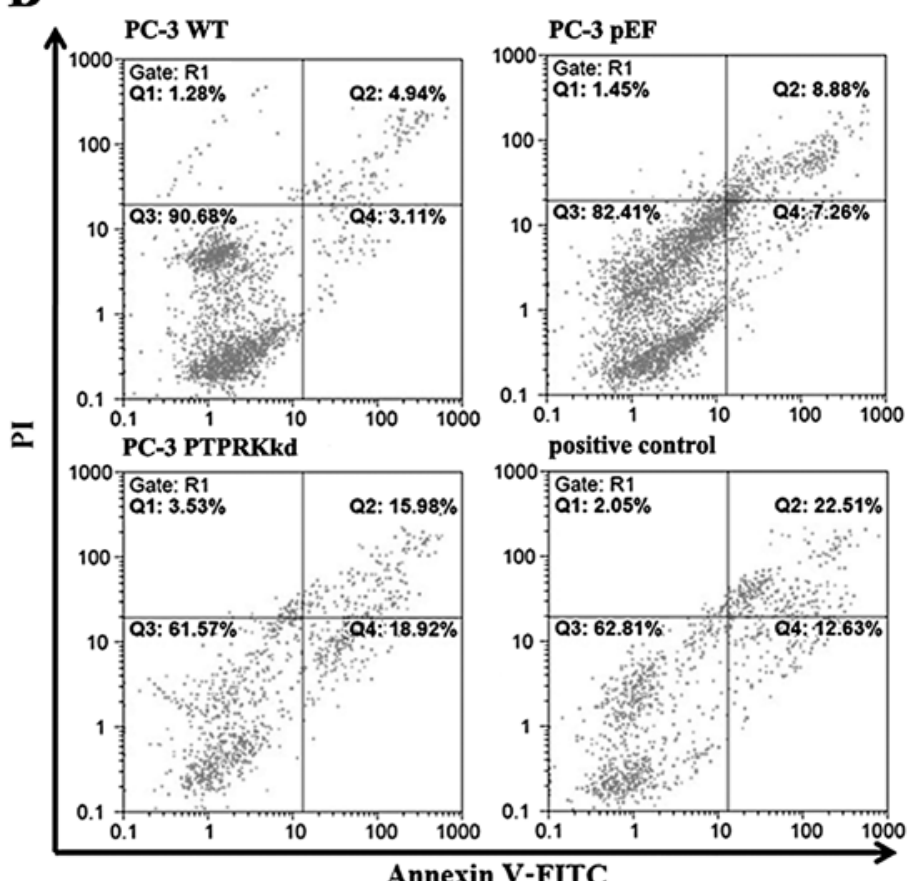

Figure 3. The effects of PTPRK knockdown on cell growth. (A) Knockdown of PTPRK decreased the in vitro growth of PC-3 cells. (B) The apoptotic population in PC-3 $3^{\text {PTPRKkd }}$ cells was increased after 72-h incubation. Segment Q1 of the quadrants refers to non-specific PI staining which are viable cells; Q2 indicates late apoptotic and necrotic cells stained with both Annexin V and PI; Q3 includes healthy and viable cells with low staining of both PI and Annexin V; and Q4 early apoptotic cells with high Annexin V, but low PI staining. Apoptotic index refers to total apoptotic population including both late apoptotic cells (Q2) and early apoptotic cells (Q4). The positive control was PC-3 cells treated with $\mathrm{H}_{2} \mathrm{O}_{2} \cdot{ }^{* * *} \mathrm{p}<0.001$.

(Fig. 5). No effect on the expression of c-Myc was observed in the PC-3 $3^{\text {PTPRKkd }}$ cells compared with the control.

The role of JNK in PTPRK knockdown-associated apoptosis. The mitogen-activated protein kinase (MAPK) pathway is the major signalling pathway involved in cellular proliferation and it affects both apoptosis and the cell cycle. As knockdown of PTPRK has been shown to impact apoptosis, its effect on the expression and activations of $\mathrm{p} 38$, JNK and ERK in PC-3 cells was analysed. The PC-3 $3^{\text {PTPRKkd }}$ cell showed a similar level of protein expression overall in p38, JNK and ERK compared with their pEF control. Furthermore, levels of phosphorylated p38, JNK and ERK were analysed using immunoprecipitation and western blot analysis. A marked increase active p-JNK (Tyr) was seen in the PTPRK knockdown cells and an increased level of active p38, suggesting that JNK and p38 may play a role in the regulation of apoptosis in the PC-3 $3^{\text {PTPRKkd }}$ cells (Fig. 6A).
Additionally, PC-3 cells were treated with p38 and JNK inhibitors for $72 \mathrm{~h}$ and analysed for apoptosis using flow cytometry. The PC- $3^{\mathrm{pEF}}$ control cells showed no effect on treatment of cells with p38 and JNK inhibitors, but apoptosis of untreated PC $-3^{\text {PTPRKkd }}$ cells $(26.70 \pm 2.87 \%)$ was dramatically increased compared with PC-3 $3^{\mathrm{pEF}}$ cells $(14.61 \pm 1.74 \%), \mathrm{p}<0.001$. The PC- $3^{\text {PTPRKkd }}$ cells treated with p38 inhibitor $(26.75 \pm 4.80 \%)$ exhibited similar apoptotic levels to the untreated cells $(13.36 \pm 2.69 \%)$. However, the PC-3 $3^{\text {PTPRKkd }}$ cells treated with JNK inhibitor $(18.76 \pm 4.28 \%)$ exhibited significant reduction of apoptotic cells compared with untreated PC-3 $3^{\text {PTPRKkd }}$ cells, $\mathrm{p}<0.001$ (Fig. 6B). The addition of the p38 inhibitor did not inhibit the effect on apoptosis, suggesting that p38 was unlikely to be involved. However, the addition of the JNK inhibitor diminished the apoptotic effect of PTPRK knockdown. This suggests that PTPRK knockdown may utilise a signalling pathway via JNK to impact apoptosis thereby inhibiting cell proliferation. 
A

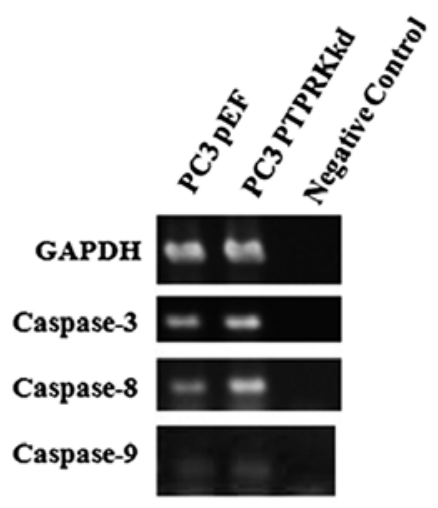

C

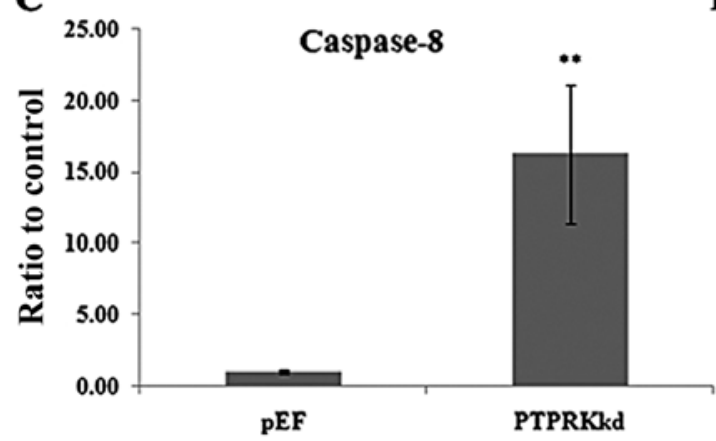

E

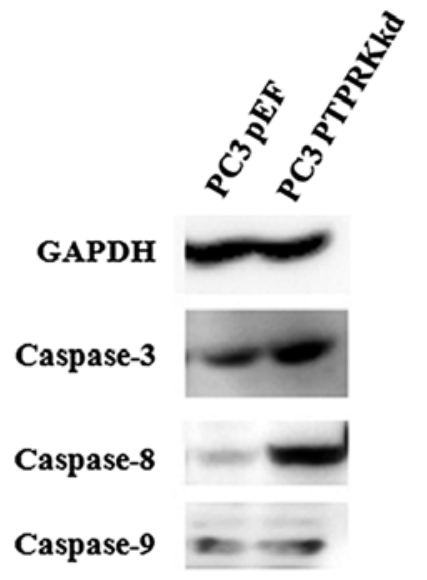

G

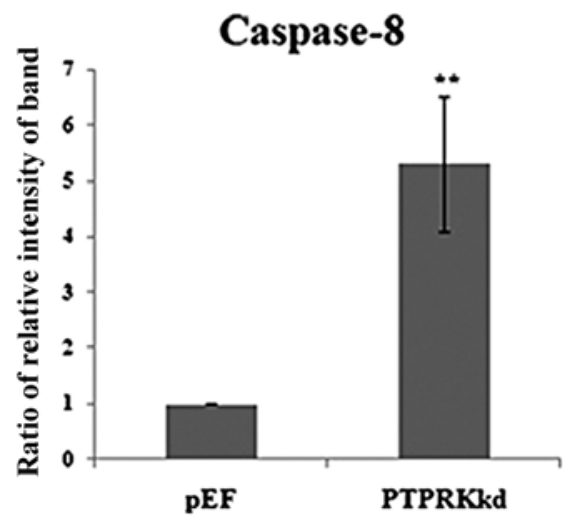

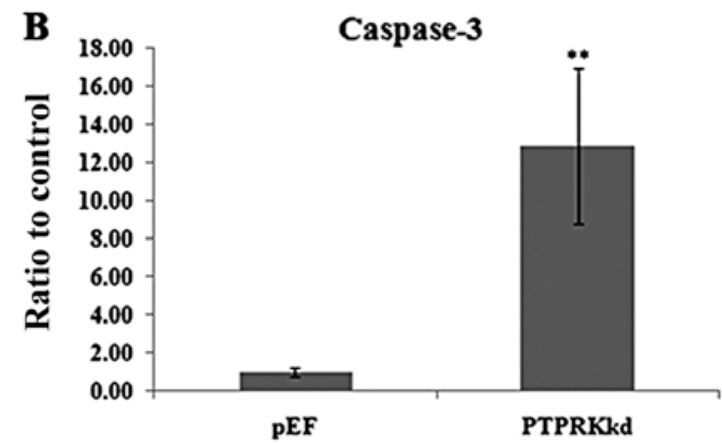

D

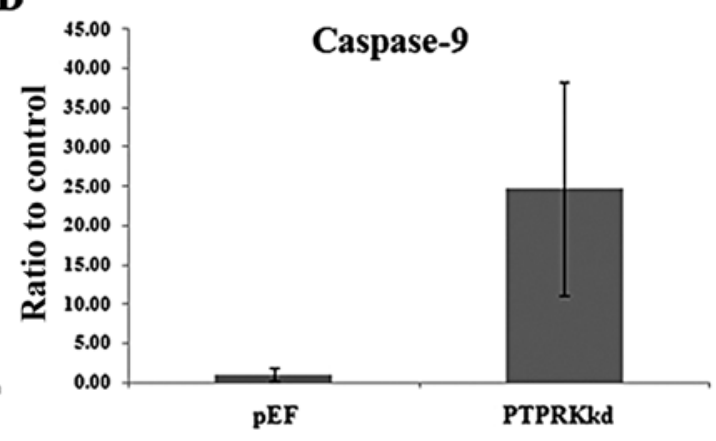

F

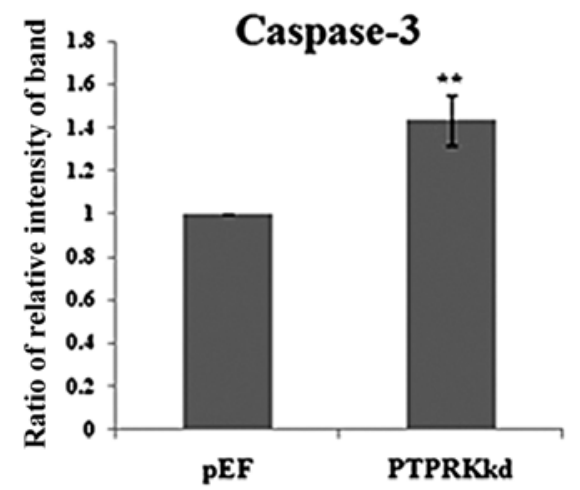

$\mathbf{H}$

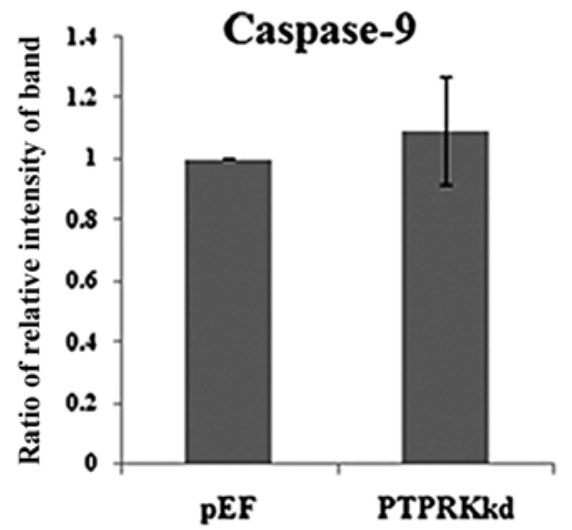

Figure 4. The effect of PTPRK knockdown on caspase-3, -8 and -9 expressions in both mRNA and protein levels. (A) PCR results of caspase-3, -8 and -9 mRNA expressions. Expression of (B) caspase- 3 and (C) caspase- 8 was increased in PC- $3^{\text {PTPRKkd }}$ cells vs. PC- $3^{\text {pEF }}$ control cells using Q-PCR. However, there is no significant difference of (D) caspase-9 seen in PC-3 $3^{\text {PTPRKkd }}$ cells. (E) The protein expression of caspase-3 and -8 was also increased in PC- $3^{\text {PTPRKkd }}$ cells, no similar change was seen in the caspase- 9 expression. Intensity of the bands from three western blots was analysed using Image J software for (F) caspase-3, (G) caspase- 8 and $(\mathrm{H})$ caspase-9. The bar graphs show quantification of three western blots of each molecule. The intensity shown is integrated band intensity (intensity $\mathrm{x}$ area) and was normalised against the corresponding GAPDH signal. ${ }^{* *} \mathrm{p}<0.01$. 

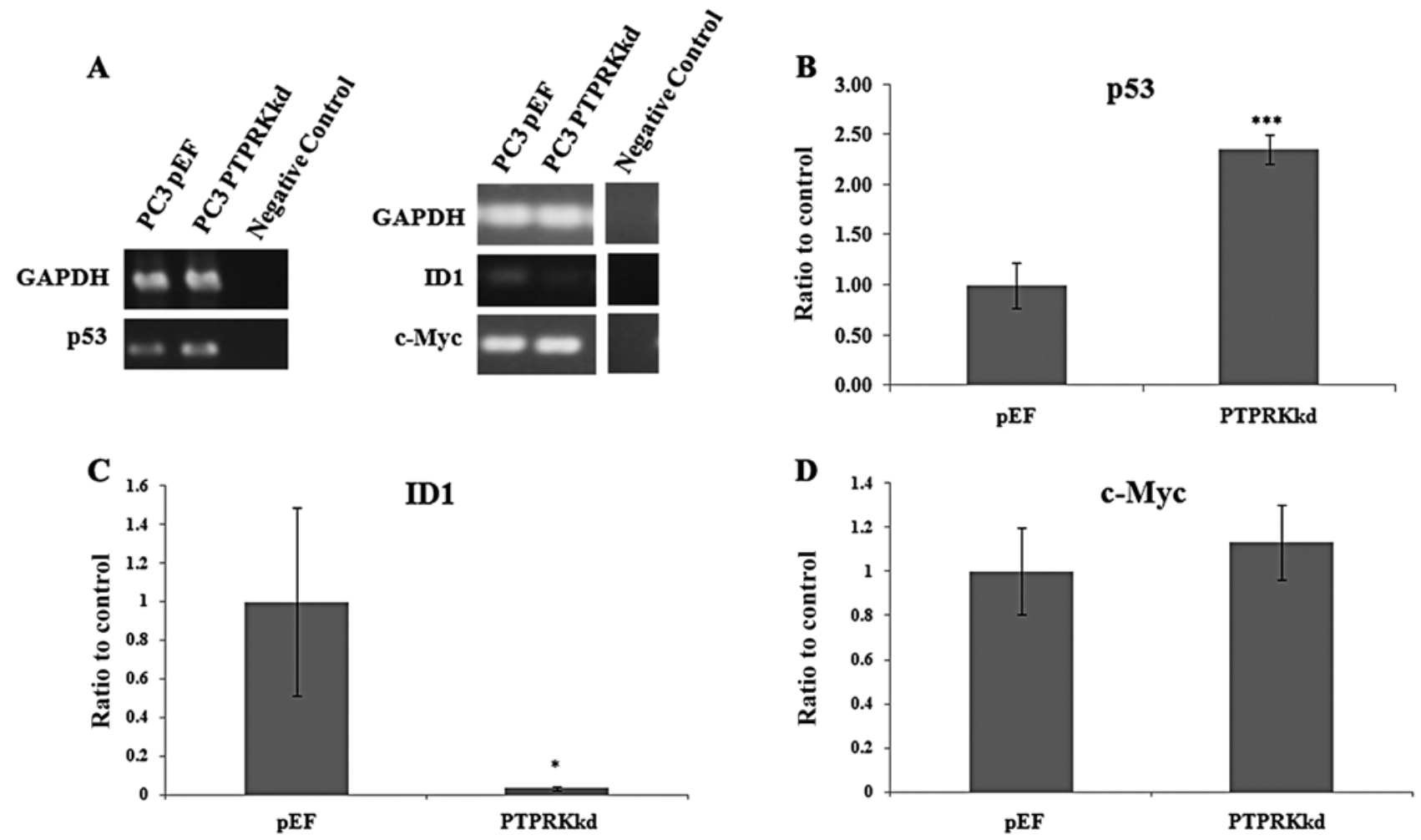

Figure 5. The effect of PTPRK knockdown on p53, ID1 and c-Myc expressions. (A) PCR results of p53, ID1 and c-Myc, demonstrated that expression of p53 was increased and expression of ID1 was decreased in PC-3 $3^{\text {PTPRKkd }}$ cells compared with their controls. (B) Expression of p53 was increased in PC-3 ${ }^{\text {PTPRKkd }}$ cells vs. PC-3 $3^{\mathrm{pEF}}$ cells control using Q-PCR. (C) Expression of ID1 was decreased in PC-3 ${ }^{\text {PTPRKkd }}$ cells vs. PC- $3^{\mathrm{pEF}}$ cells using Q-PCR. (D) There is no significant difference in c-Myc expression of PC-3 ${ }^{\text {PTPRKkd }}$ cells using Q-PCR. Q-PCR result was normalised with GADPH expression. " $p<0.05$ and ${ }^{* * * *} \mathrm{p}<0.001$.

\section{Discussion}

PTPRK is a poorly studied protein phosphatase in the field of cancer progression. It has been indicated that PTPRK is a potential tumour suppressor in primary lymphoma of central nervous system and associated with colorectal cancer and pancreatic islet tumours (12-15). No study has attempted to define the function of PTPRK in prostate cells and its potential involvement in cancer metastasis.

In this study, we demonstrate the presence of PTPRK expression in 7 prostatic cell lines and 1 human fibroblast cell line. The cell lines have extensively been used as models for in vitro studies on prostate cancer. Its expression in tissues shows that PTPRK is more highly expressed in prostate cancer tissues compared to normal prostate tissue samples in both mRNA and protein levels. According to the mRNA levels of PTPRK, its expression appears at a similar level of that seen in prostate cell lines. The only exception was PNT-1A which had lower PTPRK expression. However, the current assessment of PTPRK expression in prostate cancer is limited and not sufficient to reach any solid conclusion. Hopefully, the implication of PTPRK in disease development and progression of prostate cancer can be elucidated by further investigations using a large clinical cohort of prostate cancer.

PTPs have also been shown to exhibit different effects on tumour apoptosis (16). PTPRK has been shown to be capable of inhibiting the growth of prostate cancer cells through endogenous alteration of expression. In general, cell popula- tion is controlled by the regulation and balance between cell proliferation (cell cycle) and cell death (necrosis and apoptosis). PTPRK knockdown promoted apoptosis in prostate cancer cells and suppressed in vitro growth.

Moreover, apoptosis was associated with the activation of caspase- $-3,-8$ and -9 , and an increasing Bax:Bcl-2 ratio, followed by release of cytochrome $c$ (17). Caspase-3 is a key molecule in the late stage of apoptosis; caspase- 8 normally is activated by extrinsic receptor-mediated pathway; and caspase- 9 is activated by intrinsic mitochondrial-mediated pathway (18). In order to analyse how these molecules were affected in PC- $3^{\text {PTPRKkd }}$ cells, the expression of caspase- $3,-8$ and -9 was analysed using PCR, Q-PCR and western blot analysis. There was a significant increase in both caspase-3 and -8 expressions, but not in caspase-9 following the knockdown of PTPRK. This result showed that the increased apoptosis in PC-3 $3^{\text {PTPRKkd }}$ cells was related with extrinsic signalling rather than mitochondrial signalling.

Most PTPs play a role in promoting apoptosis. For example, PTP1B has been reported to play a role in the activation of MAPKs. PTP1B activates JNK and p38 pathways via inositol-requiring kinase 1 (IRE1) signalling and lack of PTP1B resulted in decreased levels of ER-induced apoptosis $(5,6)$. However, SHP-1 (PTPN6) dephosphorylates TrkA which in turn inhibits NGF-mediated PLC $\gamma 1$ and Akt phosphorylation, reducing the TrkA survival signal (19). In contrast, osteoclastic PTP (PTP-oc) has been reported to promote c-Src-mediated activation of NF- $\mathrm{KB}$ and JNK leading to protection from apoptosis (20). In this study, the reduction of PTPRK expression 


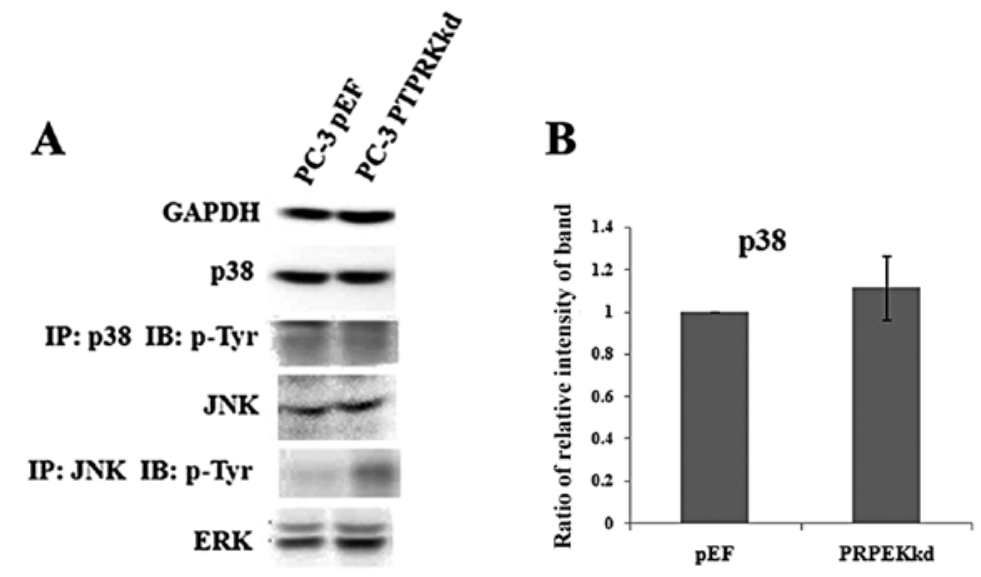

IP: ERK IB: p-Tyr

\section{C}

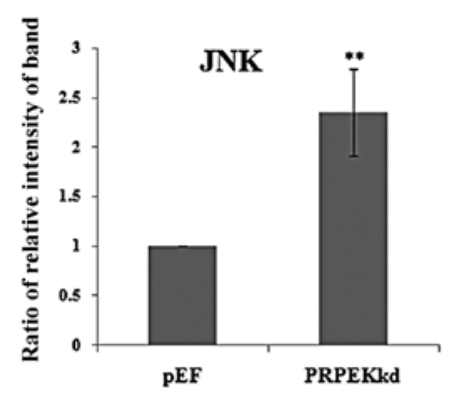

D

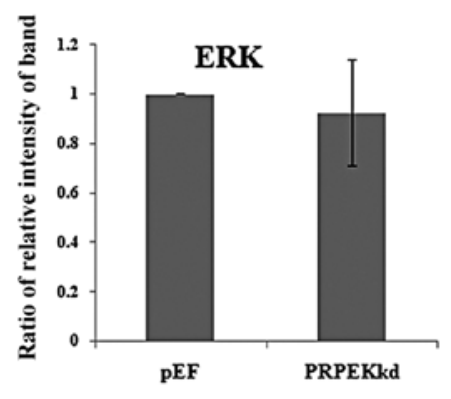

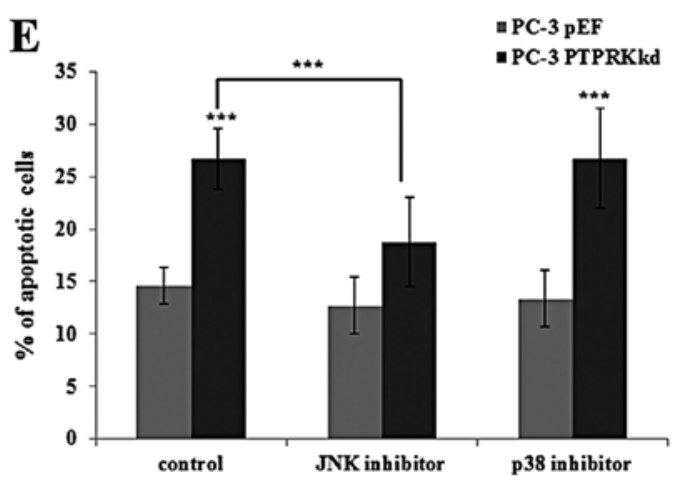

\begin{tabular}{|c|c|c|c|c|c|c|}
\hline PC-3 $(\mathrm{n}=4)$ & $\mathrm{pEF}$ & $\begin{array}{c}\mathrm{pEF} \\
\text {-JNK inhibitor }\end{array}$ & $\begin{array}{c}\mathrm{pEF} \\
+\mathrm{p} 38 \text { inhibitor }\end{array}$ & PTPRKkd & $\begin{array}{c}\text { PTPRKkd } \\
\text {-JNK inhibitor }\end{array}$ & $\begin{array}{c}\text { PTPRKks } \\
+\mathrm{p} 38 \text { inhibitor }\end{array}$ \\
\hline Mean & 14.60525 & 12.6925 & 13.36425 & 26.70083 & 18.76083 & 26.7525 \\
\hline SD & 1.738993 & 2.706835 & 2.69211 & 2.872415 & 4.27612 & 4.798424 \\
\hline
\end{tabular}

Figure 6. The effect of PTPRK knockdown on the MAPK pathway. (A) The overall expression of p38, JNK, and ERK in PC-3 ${ }^{\text {PTPRKkd }}$ cells showed similar protein levels compared with their pEF controls. In addition, there is no change in active p38 or p-ERK; however, the active p-JNK was increased significantly compared with the PC- $3^{\mathrm{pEF}}$ cells. Relative intensity of bands from three western blots was analysed using Image J software for (B) p38, (C) JNK and (D) ERK. (E) PC-3 $3^{\text {PTPRKd }}$ cells treated with JNK inhibitors exhibited a significantly decreased apoptotic population compared with untreated control, p<0.001. There was no change in apoptotic effect induced by PTPRK knockdown seen in the cells treated with the p38 inhibitor. The bar graph demonstrates the mean apoptotic percentage of four independent experiments. ${ }^{* *} \mathrm{p}<0.01$ and ${ }^{* * * *} \mathrm{p}<0.001$.

in PC-3 cells shows promotion of apoptosis and involvement of MAPK signalling pathway. The tyrosine phosphorylation of JNK was increased in PC-3 $3^{\text {PTPRKkd }}$ cells and promotion of apoptosis in PC- $3^{\text {PTPRKkd }}$ cells was diminished after treating cells with the JNK inhibitor (SP600125). These data suggest that PTPRK downregulates apoptosis in prostate cancer cells by suppressing the JNK pathway.

We also investigated other apoptosis-related molecules such as p53, ID1 and c-Myc. These molecules play crucial roles in the regulation of cell proliferation. Expression of c-Myc in tumours helps cancerous cells pass through check-points and progress to the G2/M phase of the cell cycle. In addition, expression of ID1 activates NF- $\mathrm{B}$, which then induces Bcl-2 to inhibit apoptosis. In contrast to these anti-apoptotic factors, p53 acts as a promoter of apoptosis. Furthermore, mRNA expression of p53 was increased and expression of ID1 was decreased. p53 regulates both cell cycle and apoptosis. There are some reports that show that expression of p53 is associated with the induc- 
tion of apoptosis in different cancer cells (21). p53 silencing was able to suppress cadmium-induced apoptosis in prostate cells (22). Additionally, activation of JNK can also upregulate p53 expression, leading to the accumulation of Bax which induces cell apoptosis in HeLa cells (23). In contrast, ID1 expression increases $\mathrm{NF}-\kappa \mathrm{B}$ expression which is associated with the anti-apoptotic pathway. NF- $\kappa \mathrm{B}$ activates $\mathrm{Bcl}-2$ to initiate the mitochondrial mediated anti-apoptotic effect and also activates XIAP to inhibit the activities of caspase-3 and -9 (24,25). These results indicate a complex network affected by PTPRK which participates in the coordination of cellular functions, making further investigations into the protein interactions between PTPRK and the network protein an interesting area to explore in future.

PTPRK knockdown resulted in increased apoptosis leading to the inhibition of in vitro growth of prostate cancer cells. PTPRK is a key factor in coordinating apoptosis via regulation of the MAPK pathways, in particular the JNK pathway in prostate cancer cells. Collectively, it is suggested that PTPRK may a key factor to be included in the signature of diagnosis and prediction of prostate cancer, and has great potential in the guidance of personalised treatment of malignancies.

\section{Acknowledgements}

The authors would like to thank Cancer Research Wales for their support.

\section{References}

1. Wallach D, Kang TB and Kovalenko A: The extrinsic cell death pathway and the elan mortel. Cell Death Differ 15: 1533-1541, 2008.

2. Wajant $\mathrm{H}$ and Scheurich P: TNFR1-induced activation of the classical NF- $\kappa$ B pathway. FEBS J 278: 862-876, 2011

3. Halle M, Liu YC, Hardy S, et al: Caspase-3 regulates catalytic activity and scaffolding functions of the protein tyrosine phosphatase PEST, a novel modulator of the apoptotic response. Mol Cell Biol 27: 1172-1190, 2007.

4. Akasaki Y, Liu G, Matundan HH, et al: A peroxisome proliferator-activated receptor-gamma agonist, troglitazone, facilitates caspase- 8 and -9 activities by increasing the enzymatic activity of protein-tyrosine phosphatase-1B on human glioma cells. J Biol Chem 281: 6165-6174, 2006.

5. Gu F, Nguyen DT, Stuible M, Dube N, Tremblay ML and Chevet E: Protein-tyrosine phosphatase 1B potentiates IRE1 signaling during endoplasmic reticulum stress. J Biol Chem 279: 49689-49693, 2004

6. Sangwan V, Paliouras GN, Cheng A, Dube N, Tremblay ML and Park M: Protein-tyrosine phosphatase 1B deficiency protects against Fas-induced hepatic failure. J Biol Chem 281: 221-228, 2006.
7. Gupta S, Radha V, Sudhakar C and Swarup G: A nuclear protein tyrosine phosphatase activates p53 and induces caspase-1-dependent apoptosis. FEBS Lett 532: 61-66, 2002.

8. Radha V, Sudhakar C and Swarup G: Induction of p53 dependent apoptosis upon overexpression of a nuclear protein tyrosine phosphatase. FEBS Lett 453: 308-312, 1999.

9. Jiang WG, Watkins G, Fodstad O, Douglas-Jones A, Mokbel K and Mansel RE: Differential expression of the CCN family members Cyr61, CTGF and Nov in human breast cancer. Endocr Relat Cancer 11: 781-791, 2004.

10. Zuker M: Mfold web server for nucleic acid folding and hybridization prediction. Nucleic Acids Res 31: 3406-3415, 2003.

11. Jiang WG, Davies G, Martin TA, et al: Targeting matrilysin and its impact on tumor growth in vivo: the potential implications in breast cancer therapy. Clin Cancer Res 11: 6012-6019, 2005.

12. Cady FM, O'Neill BP, Law ME, et al: Del(6)(q22) and BCL6 rearrangements in primary CNS lymphoma are indicators of an aggressive clinical course. J Clin Oncol 26: 4814-4819, 2008.

13. Nakamura M, Kishi M, Sakaki T, et al: Novel tumor suppressor loci on 6q22-23 in primary central nervous system lymphomas. Cancer Res 63: 737-741, 2003.

14. Starr TK, Allaei R, Silverstein KA, et al: A transposon-based genetic screen in mice identifies genes altered in colorectal cancer. Science 323: 1747-1750, 2009.

15. Lu J, Li Q, Donadel G, Notkins AL and Lan MS: Profile and differential expression of protein tyrosine phosphatases in mouse pancreatic islet tumor cell lines. Pancreas 16: 515-520, 1998.

16. Julien SG, Dube N, Hardy S and Tremblay ML: Inside the human cancer tyrosine phosphatome. Nat Rev Cancer 11: 35-49, 2011.

17. Halle M, Tremblay ML and Meng TC: Protein tyrosine phosphatases: emerging regulators of apoptosis. Cell Cycle 6: 2773-2781, 2007.

18. Lee ST, Wong PF, Cheah SC and Mustafa MR: Alpha-tomatine induces apoptosis and inhibits nuclear factor-kappa B activation on human prostatic adenocarcinoma PC-3 cells. PLoS One 6: e18915, 2011.

19. Marsh HN, Dubreuil CI, Quevedo C, et al: SHP-1 negatively regulates neuronal survival by functioning as a TrkA phosphatase. J Cell Biol 163: 999-1010, 2003.

20. Amoui M, Sheng MH, Chen ST, Baylink DJ and Lau KH: A transmembrane osteoclastic protein-tyrosine phosphatase regulates osteoclast activity in part by promoting osteoclast survival through c-Src-dependent activation of NFkappaB and JNK2. Arch Biochem Biophys 463: 47-59, 2007.

21. Pietsch EC, Sykes SM, McMahon SB and Murphy ME: The p53 family and programmed cell death. Oncogene 27: 6507-6521, 2008.

22. Aimola P, Carmignani M, Volpe AR, et al: Cadmium induces p53-dependent apoptosis in human prostate epithelial cells. PLoS One 7: e33647, 2012.

23. Cao H, Feng Q, Xu W, et al: Genipin induced apoptosis associated with activation of the c-Jun NH2-terminal kinase and p53 protein in HeLa cells. Biol Pharm Bull 33: 1343-1348, 2010.

24. Ling MT, Wang X, Ouyang XS, Xu K, Tsao SW and Wong YC: Id-1 expression promotes cell survival through activation of NF-kappaB signalling pathway in prostate cancer cells. Oncogene 22: 4498-4508, 2003.

25. Peng X, Wang Y, Kolli S, et al: Physical and functional interaction between the ID1 and p65 for activation of NF- $\kappa$ B. Am J Physiol Cell Physiol 303: C267-C277, 2012. 